\title{
Through the looking glass: probing the nucleus using accelerated radioactive beams
}

\author{
P.A. Butler ${ }^{1, *}$ \\ ${ }^{1}$ PH-ISOLDE, CERN, CH - 1211 Geneva 23, Switzerland
}

\begin{abstract}
Through the advent of post-accelerated beams of radioactive nuclei, probing nuclear properties of exotic nuclear species is now possible. Recent results from the new European radioactive ion beam facilities will be presented together with the prospects offered by the planned facilities such as SPIRAL2 and HIE-ISOLDE. The current ideas for the "third generation" radioactive ion beam facility EURISOL will also be briefly presented.
\end{abstract}

*permanent address: Oliver Lodge Laboratory, University of Liverpool, Liverpool L69 7ZE, UK 


\section{INTRODUCTION}

There are two types of radioactive ion beam (RIB) facilities: ISOL and 'in-flight' (or fragmentation) facilities. In the ISOL case, a beam of particles - which can be protons, neutrons, light ions or heavy ions from a so-called 'driver' accelerator - impinges on a thick target in which the radioactive nuclei are produced at rest. They then diffuse out of the target and effuse into an ion source, where they are ionised, which allows them to be extracted and accelerated. These ions can then either be transported at very low energy towards an experimental area or be post-accelerated in order to induce nuclear reactions. In an in-flight facility, a high-energy primary beam is fragmented on a thin target, and the resulting fast radioactive nuclei are selected by a fragment-separator and transported to the experimental area. No post-acceleration is necessary. The main advantages of an ISOL facility are very high secondary-beam intensities for many species (so long as their lifetime is not too short compared to the diffusion/effusion time) and beam qualities (resolution, purity and emittance), which are comparable with those of stable beams. Thus, they are well adapted to

highly elaborate experimental approaches. The fragmentation technique is most interesting (a) for very short-lived nuclei in the vicinity of the drip-lines and/or (b) for RIB production at very high energies, for which the construction of a post-accelerator would become prohibitively costly. The emittance of beams produced in-flight is poor, but storing and cooling techniques can be applied which allow for highly efficient and highly sensitive experiments, at the expense once again of the intensities of very short-lived species. The two techniques are thus very complementary.

The ISOL technique to produce radioactive ion beams has been pioneered by several European laboratories: CERN (ISOLDE and REX-ISOLDE), Louvain-la-Neuve (CRC), and GANIL (SPIRAL). These first-generation ISOL-based facilities have successfully demonstrated the feasibility of RIB production and have produced many new scientific results. The next, mid-term ISOL-based RIB facilities being constructed or in advanced stages of planning in Europe are at CERN (upgrade of REX-ISOLDE), INFN LNS (EXCYT), INFN Legnaro (SPES), MLL Munich (MAFF) and GANIL (SPIRAL2).

This paper will describe in some detail one of the present RIB facilities, REX-ISOLDE, and then outline how Europe will develop new RIB facilities in the future. Examples of applications to nuclear structure studies will also be given for both existing and planned 
facilities.

\section{REX-ISOLDE}

In the last few years the Radioactive beam EXperiment REX-ISOLDE has been operational and has been used to test new concepts of post acceleration of radioactive ions as well as use the accelerated beams for experiments in nuclear physics, astrophysics and solid state physics. REX [1] makes use of the large variety of radionuclides that have been extracted from the on-line mass separator ISOLDE. In order to use these beams significant developments in beam cooling and charge breeding were required, as whereas the output of the ISOLDE primary mass separator is a quasi-DC beam (the intensity varies between the $1.2 \mathrm{~s}$ primary proton pulses according to the release characteristics and the nuclide half-life) having charge state $1^{+}$, the low duty-factor post accelerator requires multiply-charged (A/q $<4.5)$ pulsed beams.

The first stage of the preparation of the ISOLDE beam is to continuously inject the beam from the mass separator into REXTRAP [2] where it is cooled, accumulated and extracted as bunches. The accumulation and cooling is achieved by collisions with a buffer gas. In the collisions the loss of energy of the ion enables it to be trapped within a potential barrier. The barrier is reduced every $20 \mathrm{~ms}$ so that the accumulated ions are ejected as a $20 \mu \mathrm{s}$ pulse. The transverse emittance of the beam is also reduced by the cooling procedure. This is important because the next stage, REXEBIS [3] (REX Electron Beam Ion Source), has a small acceptance ( $3 \mathrm{~mm} \mathrm{mrad})$. REXEBIS employs a $500 \mathrm{~mA} 5 \mathrm{keV}$ electron beam that is focussed to a high current density $\left(250 \mathrm{~A} / \mathrm{cm}^{2}\right)$ by a $2 \mathrm{~T}$ axial magnetic field. The ion beam is confined longitudinally by applied electric potentials and undergoes stepwise ionization via electron collisions until its mean charge state has the desired value. For A $<50$ the breeding time in REXTRAP to reach $\mathrm{A} / \mathrm{q}=3-4$ is around $20 \mathrm{~ms}$. For ${ }^{133} \mathrm{Cs}$ ions the breeding time is $80 \mathrm{~ms}$.

The yield of the radioactive isotopes from ISOLDE can be several orders of magnitude lower than the amount of residual gas ions from $\mathrm{C}, \mathrm{N}, \mathrm{O}$ and $\mathrm{Ar}$ coming out of the EBIS. Therefore, a mass separator is employed with a q/A-resolution of about 1/150 which is sufficient to select the highly charged rare radioactive ions from rest-gas contaminants.

In the first stage of the REX-ISOLDE linac the ions are accelerated from $5 \mathrm{keV} / \mathrm{u}$ to 300 
$\mathrm{keV} / \mathrm{u}$ by a 4-rod RFQ. In order to match the beam from the RFQ into the acceptance of the next stage IH (Interdigital-H-type)-structure $(0.8-1.2 \mathrm{MeV} / \mathrm{u})$, a section consisting of two magnetic quadrupole triplet lenses and a rebuncher is required. The high-energy section $(0.8-3.0 \mathrm{MeV} / \mathrm{u})$ of the linac consists of three 7-gap resonators and a 9-gap IH structure. Each resonator has a single resonance structure, which consists of a copper half shell and three arms attached to both sides of the shell. The 7-gap linac structures are operated at 101.28 MHz, while the 9-gap IH structure operates at a frequency of $202.56 \mathrm{MHz}$.

REX has already accelerated several species of radioactive ions, e.g. 10,000 ions/s of ${ }^{124} \mathrm{Cd}$ with efficiency of $\sim$ percent of the ion source yield. In 2003 REX-ISOLDE was integrated into the standard operation of CERN facilities.

\section{A. properties of neutron rich nuclei studied at REX}

One of the most interesting topics in nuclear structure research far from stability is the evolution of shell structure under the extreme conditions of large N-Z-asymmetry and weak binding, where the spin-orbit force is weakened and the standard shell ordering is modified.

For nuclei with $\mathrm{Z} \approx 12$ and $\mathrm{N} \approx 20$ the filling of the $\mathrm{f}_{\frac{7}{2}}$ intruder orbits causes an inversion of the standard shell ordering and hence the breakdown of the $\mathrm{N}=20$ shell closure. The high energy of the $2^{+}$state in the $\mathrm{N}=20$ nucleus ${ }^{34} \mathrm{Si}$ shows a strong shell effect at $\mathrm{Z}=14$, while for

${ }^{32} \mathrm{Mg}$ the energy drops below that of ${ }^{30} \mathrm{Mg}$ indicating a complete vanishing of the shell closure at $\mathrm{Z}=12$. The $\mathrm{B}\left(\mathrm{E} 2 ; 0_{g} \rightarrow 2_{1}\right)$ values for the $\mathrm{Mg}$ isotopes also demonstrate no indication of a shell closure at $\mathrm{N}=20$ for $\mathrm{Z}=12$. There is, however, a rather large uncertainty on the measured values and for ${ }^{30,32} \mathrm{Mg}$ different groups employing the same experimental method (intermediate-energy Coulomb excitation) have obtained different results. This might indicate that systematic uncertainties are present in these measurements, such as the presence of Coulomb-nuclear interference, or the effect of feeding from the many higher-lying states that are excited in the single-step process.

One of the first experiments H. Scheit et al. [4] employed $2.2 \mathrm{MeV} / \mathrm{u}^{30} \mathrm{Mg}$ beams that are Coulomb excited by $1 \mathrm{mg} / \mathrm{cm}^{2} \mathrm{Ni}$ foils; the $\gamma$-radiation was observed using MINIBALL [5] consisting of 8 3-module, 6-fold segmented HPGe detectors. The preliminary analysis of the data from this experiment has revealed that the value of the $\mathrm{B}(\mathrm{E} 2)$ for the $0^{+} \rightarrow 2^{+}$transition in ${ }^{30} \mathrm{Mg}$ is significantly lower than values obtained using intermediate-energy Coulex. 
The neutron rich $\mathrm{Ni}$ and $\mathrm{Zn}$ region is of interest because of the delicate interplay between single-particle properties and their interaction with the underlying core. For the $\mathrm{Ni}(\mathrm{Z}=28)$ isotopes the behaviour of the $2^{+}$state indicates a subshell closure at $\mathrm{N}=40$, whereas for $\mathrm{Zn}$ $(\mathrm{Z}=30)$ and $\mathrm{Ge}(\mathrm{Z}=32)$ no such effect is seen. An important experimental programme at REX is the systematic measurement of low-lying collectivity in neutron-rich nuclei having $\mathrm{N}=40-50$. P. Mayet et al. [6] have reported in this conference the first measurements of $\mathrm{B}\left(\mathrm{E} 2: 0_{g} \rightarrow 2_{1}\right)$ values in ${ }^{74,76} \mathrm{Zn}$; this same collaboration has recently measured this quantity in ${ }^{78} \mathrm{Zn}$.

The most neutron rich nuclei are the neutron halo nuclei such as ${ }^{11} \mathrm{Li}$. Precise information about the continuum states in the unbound ${ }^{9} \mathrm{Li}+\mathrm{n}$ system is therefore important for an understanding of the halo structure. One of the first experiments [7] using REX-ISOLDE was to investigate scattering states in ${ }^{10} \mathrm{Li}$ using the $\mathrm{d}\left({ }^{9} \mathrm{Li},{ }^{10} \mathrm{Li}\right) \mathrm{p}$ reaction at $2.36 \mathrm{MeV} / \mathrm{u}$,

in particular the $1 s_{\frac{1}{2}}$ state which should lie very low in the ${ }^{10} \mathrm{Li}$ spectrum. This experiment employed a $64 \mu \mathrm{m}$ segmented (16 x 16) Si $\Delta \mathrm{E}$ detector backed by a $1 \mathrm{~mm}$ Si detector to provide particle identification. Preliminary analysis of these data suggest that the lowest unbound states in ${ }^{10} \mathrm{Li}$ can be identified but further experiments at a higher bombarding energy are necessary.

\section{SECOND GENERATION RIB}

Europe is now embarking on second-generation radioactive-ion beam facilities that are either new projects or will build upon existing RIB facilities. The SPIRAL2 project at GANIL is based on a multi-beam driver (40 MeV deuterons or $14.5 \mathrm{MeV} / \mathrm{u}$ heavy ions) that will create intense RIB using several reaction mechanisms (fusion, fission, transfer, etc.) and extraction methods (ISOL, IGISOL, recoil spectrometers, etc.). These ions are postaccelerated in the existing CIME cyclotron to energies in the range 3 to $10 \mathrm{MeV} / \mathrm{u}$. The SPES project at LNL Legnaro has as its main aim the production of a large variety of neutron-rich isotopes from the fission of ${ }^{238} \mathrm{U}$, in uranium-carbide form. The neutrons are produced by a primary proton beam $(\sim 100 \mathrm{~kW})$ impinging on a thick ${ }^{9} \mathrm{~B}$ target. Once ionised these nuclei will be accelerated up to $15 \mathrm{MeV} / \mathrm{u}$ in the superconducting linac ALPI. The MAFF facility at Munich has been designed for the new research reactor FRM-II. It will deliver several intense beams $\left(\sim 3.10^{11} \mathrm{~s}^{-1}\right)$ of very neutron-rich fission fragments with a final 


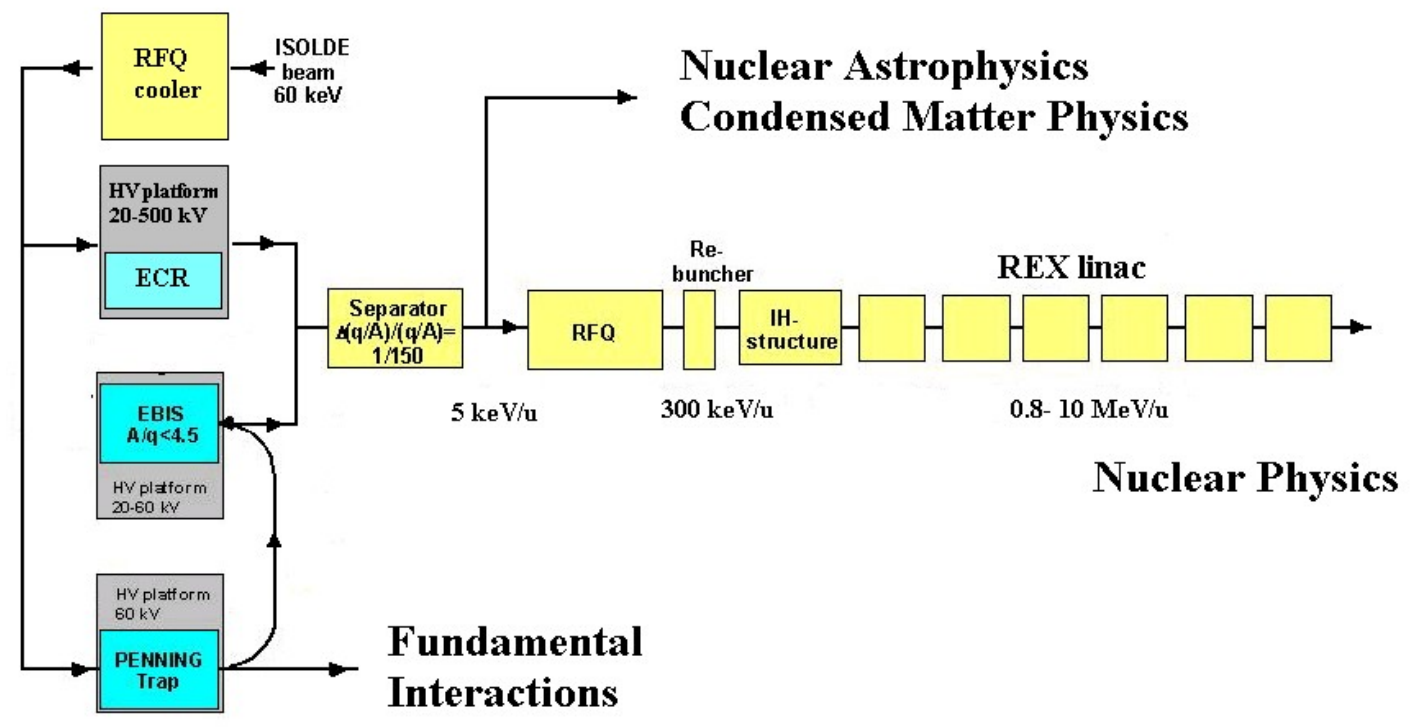

FIG. 1: Scheme of HIE-ISOLDE and its applications

energy of $30 \mathrm{keV}$ (low-energy beam) or energies between 3.7 and $5.9 \mathrm{MeV} / \mathrm{A}$ (high-energy beam).

Scheduled energy and intensity up-grades will increase the physics potential of REXISOLDE even further. The goal for the next five years will be to accelerate ions to 4.3 $\mathrm{MeV} / \mathrm{u}$ and beyond. This enhancement of the ISOLDE facility is called "High Intensity and Energy ISOLDE" or HIE-ISOLDE. The energy upgrade can be considered in two phases: (1) to increase the energy to $4.3 \mathrm{MeV} / \mathrm{u}$ by 2006 that enables certain classes of nuclear reactions to be used on a wide range of nuclei and (2) further increases in energy that will enable all reactions to be employed for all nuclei. The ISOLDE hall is being extended in order to house the additional linac cavity resonators and other instrumentation such as new recoil spectrometers for detection of fusion products. Another new facility, REX*, will provide low energy radioactive ions from a charge breeder ion source either mounted on a high voltage platform or used in conjunction with a variable-energy RFQ. This will provide beams with continuously variable energy from $<100 \mathrm{keV}$ to $>10 \mathrm{MeV}$ for astrophysics and condensed matter studies. Figure 1 shows a scheme of HIE-ISOLDE and its applications. In 
parallel with the energy upgrade, the proton beam intensity on the primary target will be increased. In the next few years, the available beam intensity from the PS booster, through the provision of $\mathrm{H}^{-}$injected beam from a new primary injector linac (LINAC 4) and a faster cycling time in the PS Booster, will be increased three-fold, resulting in a significant increase in beam intensity available to ISOLDE. The development of primary target and ion-sources that can withstand these and higher beam intensities will be made in parallel with the intensity upgrade.

\section{A. Examples of nuclear physics reach of new RIB}

A breakthrough for the study of studies of superheavy elements (SHE) came from the application of recoil detection and identification techniques $[9,10]$ to the reaction ${ }^{208} \mathrm{~Pb}\left({ }^{48} \mathrm{Ca}, 2 \mathrm{n}\right){ }^{254} \mathrm{No}$, which has a cross-section of $2 \mu \mathrm{b}$. The experimental observation $[8,9]$ of the ground-state rotational band up to spin 18 was a significant find in that it confirmed the deformed nature of the mid-shell nucleus ${ }^{254}$ No and demonstrated that the deformed shell stabilisation against fission persists to high spin. Since then, experiments have been carried out to measure the rotational properties of the even-even nuclei ${ }^{252,254}$ No and ${ }^{250} \mathrm{Fm}[10]$. These nuclei have similar values of quadrupole deformation, $\beta \sim 0.28$, and for ${ }^{252}$ No a gradual upbend of the dynamic moment of inertia is observed as compared to its isotonic and isotopic neighbours. Research efforts have also been directed to odd-mass systems that can reveal single particle properties near the Fermi surface. A severe experimental handicap in these studies arises from the internal conversion of the M1 transitions depopulating the strongly coupled rotational bands built on single particle states. In some cases that have been studied, such as ${ }^{253} \mathrm{No}$ and ${ }^{255} \mathrm{Lr}$, the gamma-ray intensities are too weak to allow the extraction of quantitative information. Gamma-ray measurements of odd mass nuclei now focus on specific cases such as ${ }^{251} \mathrm{Md}$ for which either the M1 branching is weak (cancellation of single particle and rotational g-factors) or the signature partners of the rotational band are decoupled ( $\mathrm{K}=1 / 2$ ground state). The use of radioactive beams will extend the range of nuclei that can be studied, and sufficient beam intensities of neutron rich projectiles that neighbour ${ }^{48} \mathrm{Ca}$ such as ${ }^{43} \mathrm{Ar}$ and ${ }^{46} \mathrm{~K}$ will be available from the next generation radioactive beam facilities. Another important landscape that can be studied with radioactive beams is the nuclear potential energy surface of deformed SHE. Egido and Robledo have 
predicted [11] that the second minimum in ${ }^{254}$ No becomes yrast at about spin $20-30 \hbar$. The reaction ${ }^{164} \mathrm{Dy}\left({ }^{92} \mathrm{Kr}, 2 \mathrm{n}\right){ }^{256} \mathrm{No}$ leads to an excitation energy in the residue nucleus similar to that induced by ${ }^{48} \mathrm{Ca}$, but with significantly higher angular momentum. High intensity ${ }^{92} \mathrm{Kr}$ beams will be available with the second-generation facilities SPIRAL2, SPES and MAFF.

The pairing of like nucleons in a superfluid condensate is a well known aspect of the structure of low-lying states in nuclei near stability. Heavier nuclei with equal numbers of neutrons and protons are expected to provide the first evidence for two further modes of nuclear superconductivity in which the Cooper pairs involve a neutron and a proton so that the restrictions from the Pauli principle are removed. In particular paired states of zero orbital angular momentum in which the intrinsic spins are parallel can only occur for the neutron-proton system. The best nuclei in which to search for these phenomena are the heavier $\mathrm{N}=\mathrm{Z}$ systems, where there are strong co-operative proton-neutron interactions, sufficient particles to allow large-scale correlations to develop and a high level density, allowing easy scattering of pairs between levels. The manifestation of neutron-proton pairing may appear directly in the enhancement of n-p transfer in reactions such as ${ }^{58} \mathrm{Cu}(\mathrm{d}, \alpha){ }^{56} \mathrm{Ni}$ and ${ }^{62} \mathrm{Ga}(\mathrm{d}, \alpha){ }^{60} \mathrm{Zn}$ for which EURISOL will be able to provide radioactive beams of sufficient intensity.

\section{EURISOL}

The European nuclear physics community are currently advocating the construction of two 'next generation' RIB infrastructures in Europe, one ISOL facility and one in-flight facility. The in-flight machine would arise from a major upgrade of the current GSI facility (FAIR), while EURISOL would constitute the new ISOL facility. A preliminary design study of EURISOL [12], was implemented under the auspices of the EU 5th framework program. The result is a preliminary design report which outlines a concept for a future EURISOL facility. The driver accelerator would be a super-conducting CW proton linac, of energy 1 $\mathrm{GeV}$ and power $5 \mathrm{MW}$, with additional capability of accelerating deuterons and possibly heavier ions with $\mathrm{A} / \mathrm{Q}=2$. Several target stations would be built, including a fission target with a liquid mercury converter allowing for the use of the full $5 \mathrm{MW}$ beam power, and targets receiving directly the approximately $100 \mathrm{KW}$ of proton beam for production of lighter or neutron deficient isotopes. The post accelerator would be a super-conducting heavy-ion linac 


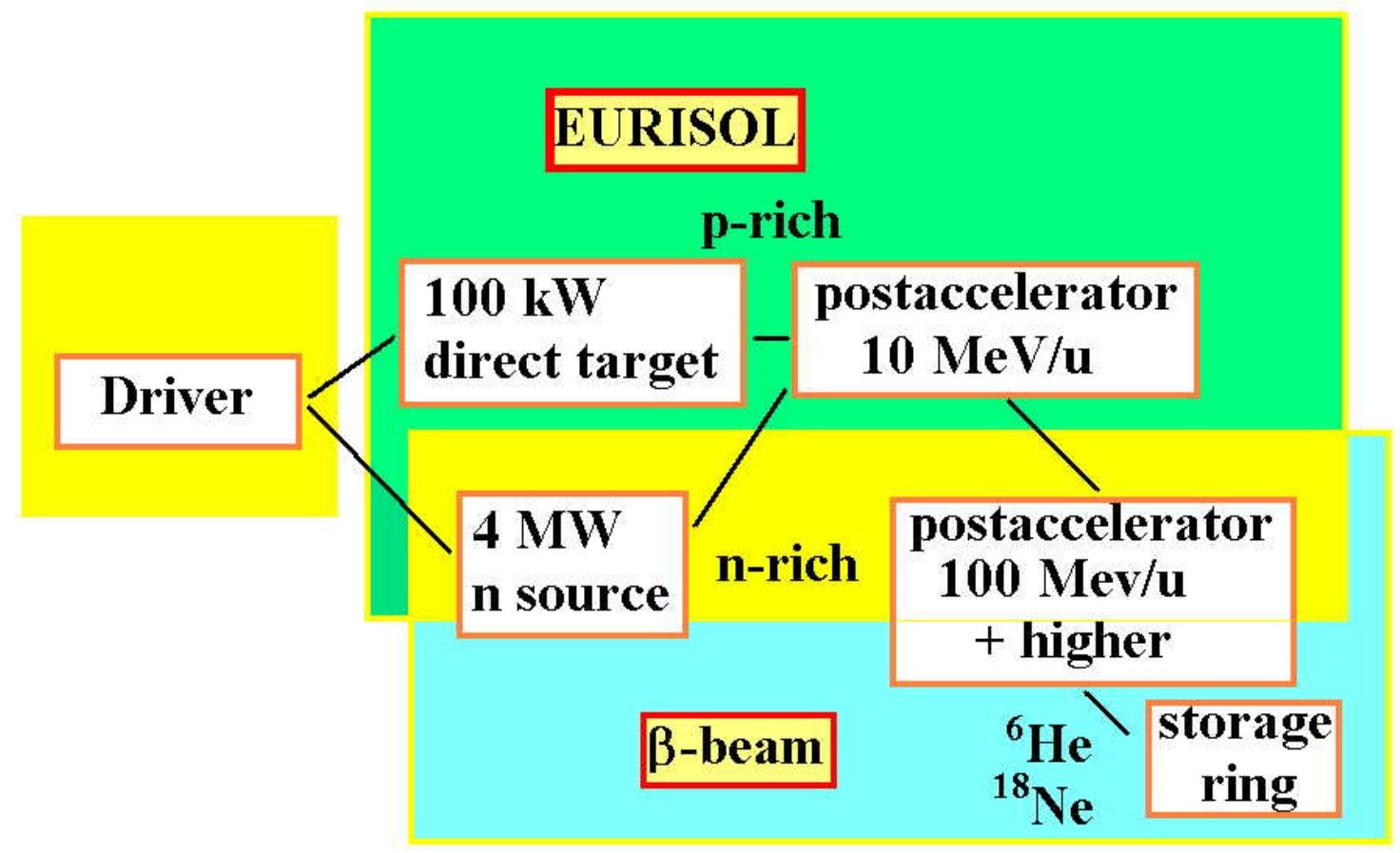

FIG. 2: Scheme of EURISOL and its synergy with the beta-beam facility

with a maximum energy of $100 \mathrm{MeV} /$ nucleon. The maximum energy is somewhat arbitrary and for a linac is defined by cost (note that higher energies could be achieved at CERN by exploiting its existing synchrotron accelerator chain or new accelerators required for the beta-beam facility, see next section).

In EURISOL there will be several experimental areas devoted to physics at different energies: physics of fundamental interactions, solid-state physics and nuclear medicine, nuclear astrophysics, nuclear structure and nuclear reaction studies. With the wide diversity of scientific disciplines and individual experiments being served by the facility, various multi-user installations (such as at the present ISOLDE) are needed, requiring the design of a beam switchyard that allows parallel operation.

Typical intensities in particles per second would be $10^{13}$ for ${ }^{132} \mathrm{Sn}, 10^{11}$ for ${ }^{56} \mathrm{Ni}, 5.10^{13}$ for ${ }^{6} \mathrm{He}$, and $5.10^{12}$ for ${ }^{18} \mathrm{Ne}$. The total cost of such a facility has been estimated as 600 $\mathrm{M}$ euros, including buildings but excluding manpower. The high-intensity of ${ }^{6} \mathrm{He}$ and ${ }^{18} \mathrm{Ne}$ beams available would make EURISOL an attractive source of $\beta$-unstable nuclei for an electron neutrino facility ('beta-beam', see next sub-section), see figure 2. 


\section{A. EURISOL Design Study}

In close contact with the nuclear physics and neutrino communities, a design study proposal (EURISOL DS) was submitted in March 2004, with the aim of performing detailed engineering oriented studies and technical prototyping work for the future EURISOL facility. This design study proposal includes 21 participating institutions from 14 European countries, as well as 21 other contributing laboratories from Europe, North America and Asia who will provide their expertise on specific technical points. The total cost of the design study would be $33 \mathrm{M}$ euros, of which $9 \mathrm{M}$ euros is requested from the EU, the remainder being provided by the participating institutions.

The work is to be subdivided in 11 tasks: physics and instrumentation, beam intensity calculations, safety and radioprotection, proton accelerator design, heavy ion accelerator design, superconducting cavity development, multi-MW target station, direct target, fission target, beam preparation, and beta-beam aspects.

Three examples of sites for EURISOL have already be identified:

(1) The European Joint Laboratory comprising INFN-Legnaro and GANIL presents the scientific case for EURISOL to the Italian and French funding agencies. The infrastructure would in this case be sited at one of these national laboratories and the two major shareholding countries would provide more than $50 \%$ of the cost of the facility. Other countries would be invited to join the consortium that would build the facility. It is expected that EURISOL construction would make use of developments at existing infrastructures (SPIRAL2, SPES).

(2) The International Organisation CERN, whose member state contributions are agreed by international treaty, hosts EURISOL. In this case EURISOL will benefit from synergies with the fixed target physics programme (e.g. neutrino beams) and requirements for the LHC upgrade which demand the construction of a new high-intensity proton injector, e.g. the Superconducting Proton Linac (SPL). The decision procedure consists of workshops followed by scientific and technical reports; CERN's Scientific Policy Committee will review these reports and make the final recommendation to the CERN Council. In this case EURISOL would benefit from existing ISOL and accelerator infrastructure. In fact CERN is currently considering the scientific case for the SPL [13]. This accelerator will provide $2.2 \mathrm{GeV}$, $11 \mathrm{~mA}$ CW proton beams for enhanced injection to the PS and thence to the LHC, and to provide intense proton beams for neutrino production and to a next generation RIB 
facility. There are a number of scenarios for neutrino production. In the Neutrino Factory the proton beam strikes a fixed target producing pions which are captured in a solenoidal channel where they decay into muons. The muons are subsequently cooled and accelerated before injection into a bow-tie or triangle shaped decay ring. A new concept for neutrino beams having strong synergies in accelerator and target technology with a RIB facility is the beta-beam [14] in which ${ }^{6} \mathrm{He}$ and ${ }^{18} \mathrm{Ne}$ radioactive ions are accelerated to a Lorentz gamma of $\approx 100$ before injection into a decay ring, thus producing a flavour-pure beam of antineutrinos and neutrinos respectively. The high duty cycle of the CERN SPL (at least $150 \mu \mathrm{s}$ available of the $2.8 \mathrm{~ms}$ pulse width, repeated at a frequency of $50 \mathrm{~Hz}$ ) provides a good match to the specification of the primary driver of EURISOL. This will give a lower peak intensity of the proton beam than the present PS-Booster ISOLDE facility (thereby increasing the target lifetime), while the average current for direct RIB production can be increased to 100 $\mu \mathrm{A}$ or more compared to the present value of $\approx 2 \mu \mathrm{A}$. The higher proton energy will give an order of magnitude increase (over that from the existing ISOLDE facility) in the formation cross-sections for the light neutron rich target fragmentation products, while operating the SPL at lower energies than the nominal 2.2 GeV permits the RIB production cross-section of the spallation products to be optimised. The high power available (4 MW) also allows the use of a spallation neutron target for production of intense beams of fission fragments in a secondary target. The driver beam will be transported to both the existing ISOLDE facility and a new target area that is located underground. Post-accelerated RIB beams of energies up to and beyond $10 \mathrm{MeV} / \mathrm{u}$ will be transported to at least three experimental areas, with possibilities to be explored of injection into the PS Booster to accelerate heavy radioactive ions to $100 \mathrm{MeV} / \mathrm{u}$ or to inject into storage rings with electrons, muons, or antiprotons.

(3) A "green-field" site could be identified in a less-favoured region of Europe, which is close to laboratories having a tradition in accelerator and nuclear physics. Examples are these are Catania, Liverpool and Valencia. In this case use would be made of European Structural Funds, and such a construction would have important regional and trans-regional technological benefits.

I am grateful to U. Bergmann, O. Niedermaier, H. Scheit, and P. Mayet for providing preliminary data, and to Y. Blumenfeld, G. Fortuna, R.-D. Herzberg, W. Gelletly, M. Lindroos, 
T. Nilsson and D. Warner for helpful discussions.

[1] D. Habs et al., Hyperfine Interactions, 129 (2000) 43

[2] G. Bollen et al., Nucl. Instrum. Meth. A, 368 (1996) 675

[3] B.H. Wolf et al., Nucl. Instrum. Meth. B, 204 (2003) 428

[4] O. Niedermaier et al. TC63b, this conference

[5] J. Eberth et al., Prog. Part. Nucl. Phys. 46 (2001) 389

[6] P. Mayet et al., P90, this conference

[7] H. Jeppesen, P126, this conference

[8] P. Reiter et al., Phys. Rev. Lett. 82 (1999) 509

[9] M. Leino et al., Eur. Phys. J. A6 (1999) 63

[10] R.-D. Herzberg, J. Phys. G: Nucl. Part Phys. 30 (2004) R123

[11] J.L. Egido and L.M. Robledo Phys. Rev. Lett. 85 (2000) 1198

[12] J. Vervier et al., http://www.ganil.fr/eurisol

[13] B. Autin et al.: Conceptual design of the SPL, a high-power superconduction $\mathrm{H}^{-}$Linac at CERN, CERN-2000-012, 2000

[14] P. Zucchelli, Phys. Lett. B, 532 (2002) 166 